\title{
Analysis of the effects and relationship of perceived handwritten signature's size, graphical complexity, and legibility with dynamic parameters for forged and genuine samples
}

\begin{abstract}
This article presents an analysis of handwritten signature dynamics belonging to two authentication groups, namely genuine and forged signature samples. Genuine signatures are initially classified based on their relative size, graphical complexity, and legibility as perceived by human examiners. A pool of dynamic features is then extracted for each signature sample in the two groups. A two-way analysis of variance (ANOVA) is carried out to investigate the effects and the relationship between the perceived classifications and the authentication groups. Homogeneity of variance was ensured through Bartlett's test prior to ANOVA testing. The results demonstrated that among all the investigated dynamic features, pen pressure is the most distinctive which is significantly different for the two authentication groups as well as for the different perceived classifications. In addition, all the relationships investigated, namely authenticity group versus size, graphical complexity, and legibility, were found to be positive for pen pressure.
\end{abstract}

Keyword: Forensic science; Document examination; Signature examination; Dynamic parameters; Perceived signature classification; Statistical hypothesis testing 